\title{
STEM CELLS
}

INTESTINAL TISSUE

GENERATED IN VITRO

Induced pluripotent stem cells have been coaxed to develop into three-dimensional intestinal tissue in vitro by researchers from Ohio, USA. "This is an important first step in generating intestinal tissue for transplantation," notes James Wells, corresponding author of the study. $\mathrm{He}$ suggests that such tissues could also be useful for screening and designing drugs with improved oral absorption.

Pluripotent stem cells can be induced to differentiate into a single cell type relatively easily, but getting them to produce all the cell types present in an organ and to replicate its complex three-dimensional architecture presents a considerable challenge. The team had previously identified molecular pathways that direct intestinal development in animal models. They then manipulated these same pathways in cultured stem cells to mimic the development of intestinal tissue during embryogenesis.

"We were stunned by how well it worked," says Wells. The team initially induced human pluripotent stem cells to form a monolayer of a single cell type, the definitive endoderm, which was then coaxed to develop into tubular hindgut by the stepwise application of various growth factors at specific time points. When cultured under conditions that promote the growth and renewal of adult intestinal epithelium, these structures developed into organoids composed of all the major intestinal cell types that also demonstrated key functional properties, including nutrient absorption and secretion of intestinal proteins.

Their three-dimensional architecture and cellular composition are "remarkably similar" to that of fetal intestine, the researchers write in Nature.

Ultimately, Wells hopes that cultured intestinal tissue could be used to treat patients with short gut syndrome resulting from necrotizing enterocolitis or surgery for Crohn's disease, IBD and cancer.

Caroline Barranco

Original article Spence, J. R. et al. Directed differentiation of human pluripotent stem cells into intestinal tissue in vitro. Nature doi:10.1038/nature09691 